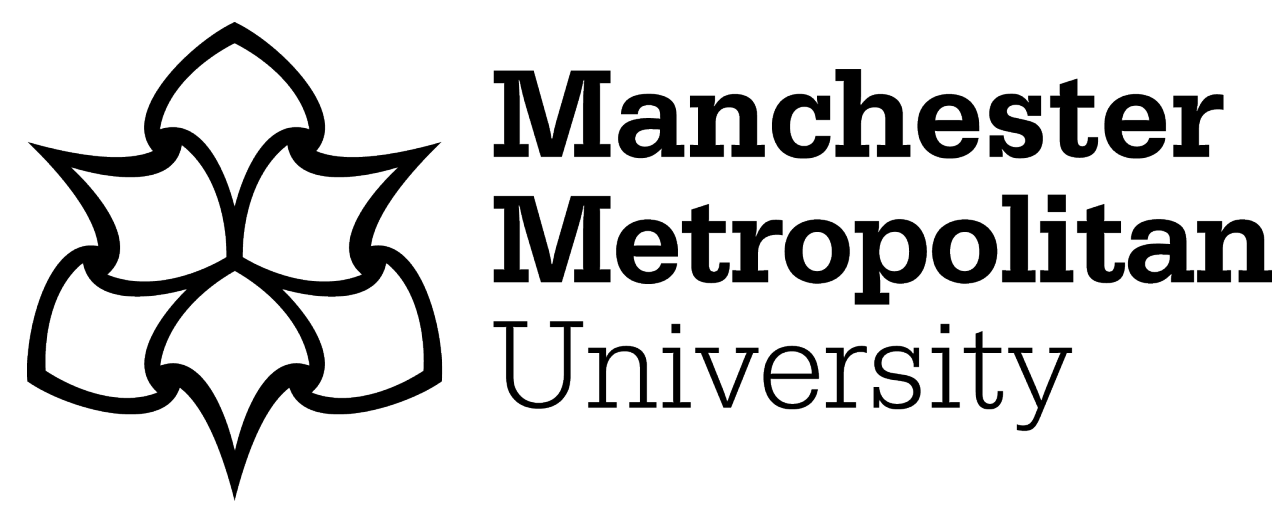

Zhang, Di, Liu, Yuanwei, Dai, Linglong, Bashir, Ali Kashif, Nallanathan, Arumugam and Shim, Byonghyo (2019) Performance Analysis of FD-NOMAbased Decentralized V2X Systems. IEEE Transactions on Communications, 67 (7). pp. 5024-5036. ISSN 0090-6778

Downloaded from: https://e-space.mmu.ac.uk/622899/ Version: Accepted Version

Publisher: Institute of Electrical and Electronics Engineers (IEEE) DOI: https://doi.org/10.1109/tcomm.2019.2904499

Please cite the published version 


\title{
Performance Analysis of FD-NOMA-based Decentralized V2X Systems
}

\author{
Di Zhang, Member, IEEE, Yuanwei Liu, Member, IEEE, Linglong Dai, Senior Member, IEEE, \\ Ali Kashif Bashir, Senior Member, IEEE, Arumugam Nallanathan, Fellow, IEEE, and Byonghyo Shim, Senior \\ Member, IEEE
}

\begin{abstract}
In order to meet the requirements of massively connected devices, different quality of services (QoSs), various transmit rates and ultra-reliable and low latency communications (URLLC) in vehicle to everything (V2X) communications, we introduce a full duplex non-orthogonal multiple access (FDNOMA)-based decentralized V2X system model. We then classify the V2X communications into two scenarios and give their exact capacity expressions. To solve the computation complicated problems of the involved exponential integral functions, we give the approximate closed-form expressions with arbitrary small errors. Numerical results indicate the validness of our derivations. Our analysis has that the accuracy of our approximate expressions is controlled by the division


the suburban and remote scenario. Numerical results manifest 1) Increasing the number of V2X device, NOMA power and Rician factor value yields better capacity performance. 2) Effect of FD-NOMA is determined by the FD self-interference and the channel noise. 3) FDNOMA has better latency performance compared to other schemes.
\end{abstract}

Index Terms-Vehicle communications, V2X, full duplex, nonorthogonal multiple access, capacity analysis.

\section{INTRODUCTION}

\section{A. Background}

There are two distinct regimes in vehicle to everything (V2X) communications, i.e., the dedicated short-range communications (DSRC) [1], [2] and the cellular-V2X (C-V2X) [3], [4].

DSRC was popular in the past decades. Recently, C-V2X

Corresponding author: B. Shim \{bshim@snu.ac.kr\}.

The work of D. Zhang and B. Shim is supported by the NRF grant funded by the Korean government (MSIP2014R1A5A1011478), the work of D. Zhang is also supported by the Zhengzhou University Startup Foundation (32210907), the National Natural Science Foundation of China (61801435), the China Postdoctoral Science Foundation (2018M633733). L. Dai is supported by the National Natural Science Foundation of China for Outstanding Young Scholars (Grant No. 61722109).

Di Zhang is with the School of Information Engineering, Zhengzhou University, Zhengzhou, 450001, China, and also with the Information System Laboratory and Department of Electrical and Computer Engineering, Seoul National University, Seoul, 08826, Korea (e-mail: di_zhang@zzu.edu.cn).

Yuanwei Liu and Arumugam Nallanathan are with the EECS, Queen Mary University of London, London, E14NS, U.K. (e-mail: \{yuanwei.liu, a.nallanathan\}@qmul.ac.uk).

Linglong Dai is with the Department of Electronic Engineering, Tsinghua University, Beijing 100084, China (e-mail: daill@tsinghua.edu.cn). Ali Kashif Bashir is with the School of Computing, Mathematics, and Digital Technology, Manchester Metropolitan University, Manchester M15 6BH, U.K. (e-mail: dr.alikashif.b@ieee.org ).

Byonghyo Shim is with the Institute of New Media and Communications and Department of Electrical and Computer Engineering, Seoul National University, Seoul, 08826, Korea (e-mail: bshim@snu.ac.kr). has received much attention with explosively growing devices connecting to the wireless networks. With the help of cellular network, C-V2X can connect more V2X devices [5], [6]; it can establish the link among vehicles, smart infrastructures and pedestrians. C-V2X operates in two modes. First, in the direct communications (DC) mode, V2X devices can directly communicate with each other. Well-known examples include vehicle to vehicle (V2V), vehicle to pedestrian (V2P) communications. Second, in the network-based communications (NC) mode, cellular base station (BS) is playing the dominant role, and the V2X devices communicate with (or with the help of) the cellular, for instance, vehicle to network (V2N), vehicle to infrastructure (V2I) communications. However, the current version of C-V2X (i.e., the long term evolution V2X (LTEV2X)) cannot fully satisfy the requirements of low latency, various quality of services (QoSs) and different transmit rates [6], [7].

In addition, the existing orthogonal frequency division multiple access (OFDMA)-basced LTE-V2X systems need orthogonality. Different from the static or non-mobility wireless communications, moving vehicle caused Doppler effect is a vital problem for OFDMA-based LTE-V2X systems [8]. As is known, carrier frequency offset (CFO) caused by the Doppler effect will lead to inter-carrier interference $(\mathrm{ICl})$ to the OFDMbased wireless communications [9]. In literature, there have been various studies to solve the CFO compensation, see, e.g., [9], [10]. However, because the oscillators can never be oscillating at the identical frequency, in OFDMA-based wireless communications, CFO side-effect always exists even for nonmoving circumstance [9].

It is noticed that besides the OFDMA, some fifth generation (5G) technologies can be used to address the issues of low latency [11], various QoS and different transmit rates in V2X communications. From the upper layer perspective, the software-defined networks (SDNs) with its centralized control plan and distributed multiple nodes are more suitable for vehicle communications. With the aid of machine learning and big data analysis, we can monitor all types of events and maintain a global network status [12]. From the physical layer perspective, under the equal frequency resources constraint, NOMA can accommodate more users comparing to the orthogonal multiple access (OMA) scheme. Besides, these users can be with different QoS requirements [13], [14]. In addition, NOMA is insensitive to CFO effect caused by moving vehicles 
because of its non-orthogonal frequency. NOMA employs the same resource block (RB) for multiple user's transmission, which can alleviate the spectrum bottleneck of wireless communications [15]-[17]. NOMA can pair users with different transmit rates for simultaneous transmission [18]-[20]. On the other hand, while simultaneously transmitting and receiving information, full duplex (FD) can provide faster speed and better spectrum efficiency (SE) performances [13]. Moreover, FD can offer reliable communications [21], which is useful for V2X applications such as navigation and emergency message broadcasting.

\section{B. Related Works and Motivations}

Some antecedent works has been done on FD-NOMA. For instance, it was found that FD-NOMA can significantly suppress the co-channel interferences and achieve better performance gains compared to half duplex NOMA (HDNOMA) and orthogonal multiple access (OMA) [22]. Analysis and simulation results in [23] demonstrated that rate region performance of FD-NOMA outperforms the one with NOMA. Analysis and simulation results in [24] indicates that FDNOMA improves the 5G's system performance compared to HD-NOMA. Based on the relaying system model, analysis and simulation results in [13] indicated that FD-NOMA outperforms HD-NOMA in terms of outage probability and ergodic sum rate in low signal to noise ratio (SNR) region, but displays an inferior performance in high SNR region.

In V2X communications, there are some existing works on NOMA-V2X and FD-V2X [25]-[27]. Based on the NOMA, the authors in [26] proposed the graph-based practical encoding and joint belief propagation (BP) decoding techniques, which can achieve any rate pair close to the capacity region. B. Di et al. in [25] employed NOMA for URLLC communications while proposing a NOMA-based mixed centralized/distributed (NOMA-MCD) scheme to reduce the resource collision. In [27], an optimal blind interference alignment scheme was proposed for the coexisting of FD and HD modes. This scheme can improve the sum rate performance in the finite SNR regime. However, most of these studies on NOMA-V2X and FD-V2X communications are based on the NC mode, which is a challenge for connecting massive V2X devices because of the cellular throughput restriction. Although the authors investigated the decentralized NOMA-V2X systems in [25], there has been no capacity analysis for such a system. To the best of our knowledge, a study investigating the impact of FDNOMA techniques on V2X systems is rare, which motivates us to develop this treatise.

\footnotetext{
${ }^{1}$ Besides C-V2X communications, there are other types of cellular communications, our work can not offload all the cellular network load
}

In literature, various channel models are used for the ergodic capacity analysis, for instance, the $\kappa-\mu$ channel model [28], [29] and the $\eta-\mu$ channel model [28]. However, obtaining the closed-form capacity expression in these channel models is difficult because of the involved infinity series operations. Authors thus employed some special conditions and methods to give the closed-form expressions, e.g., $\mu$ with positive integer values [29] and the approximate method [30]. On the other hand, the difficulty to obtain a closed-form expression with Rayleigh or Rician channel model lies in the involved exponential integral functions. In order to solve this problem, some approximate methods and algorithms have been proposed, for instance, the Swamee and Ohija method for exponential integral function [31] and the fast and accurate algorithm for generalized exponential integral function [32]. However, these methods are based on some special conditions (e.g., [32]), or with low accuracies (e.g., [31]). In this paper, we give the approximate closed-form capacity expressions for both Rayleigh and Rician channel models while taming the troublesome exponential integral functions.

In this work, we propose the FD-NOMA-based decentralized V2X system model, and also provide the capacity analysis to obtain the approximate closed-form capacity expressions with high accuracy. We try to answer the following key questions.

- Can we use one solution to meet the requirements of V2X communications? If it is not possible, what about a combination of FD-NOMA techniques?

- If the combination is feasible to satisfy the requirements of V2X communications, what about the capacity and throughput performance of the V $2 X$ systems?

- Is there any approximate expressions for the capacity expressions with arbitrary small error and low computational complexity?

\section{Contributions}

The main contributions of this work can be summarized as follows:

- The FD-NOMA-based decentralized V2X systems can partly offload the cellular network ${ }^{1}$. Compared to OFDMA, NOMA is insensitive to Doppler effect caused by moving vehicles. In addition, FD-NOMA can accommodate more users with different QoSs and transmit rates for simultaneous transmission and reception.

- Based on the system model, we derive the exact system ergodic capacity expressions and their approximate closedform expressions for both scenarios. These approximate closed-form expressions are with low computational complexity and controllable arbitrary small errors compared to the existing approximate expressions. 
Insights from our analysis has 1) the accuracy of our simplified approximate expression in urban and crowded scenario is controlled by the associated division of $\mathbf{D i t h}$ respect to the exponential integral function $\left.E_{1}(x)\right)$. 2) The accuracy of our simplified approximate expression in suburban and remote scenario is controlled by the truncation point $T$ (with respect to the exponential integral function $\left.E_{n}(x)\right)$. • It is observed from our numerical results that: 1 ) the analytical results coincide with the Monte-Carlo based simulation results perfectly, which demonstrates the validity of our derivations. 2) The system capacity increases with the increasing allocated power value, SNR and Rician factor values. 3) The FD selfinterference and the channel noise determine the effect of FD-NOMA. 4) FD-NOMA has better latency performance compared to HD-NOMA and HD-orthogonal multiple access (HD-OMA) schemes.

\section{Notations and Organization}

Notations: In this article, we use upper case boldface letters to denote matrices (e.g., A), and we use lower case boldface letters to denote vectors (e.g., a). In addition, we use $\mathbf{A}^{T}$ as the transpose of $\mathbf{A}, \mathbf{a} \cdot \mathbf{b}$ to denote the multiply by position operation for two vector $\mathbf{a}$ and $\mathbf{b}$. On the other hand, $A \leftrightarrow B$ means a transmit-receive pair with $A$ and $B$ transceivers on each side working on FD mode, $A \rightarrow B$ the transmission procedure from $A$ to $B$, vice versa.

The remainder of the paper is organized as follows. In section II, the FD-NOMA-based decentralized V2X system model is proposed. We divide the $\mathrm{V} 2 \mathrm{X}$ communications into different scenarios in this section. We analysis the system capacity of different scenarios in section III. The numerical simulations are given by section IV, and conclusion is given in section $\mathrm{V}$.

\section{The FD-NOMA-BASEd DeCENTRALIZEd V2X SyStemS}

\section{A. System Model}

The FD-NOMA-based decentralized V2X system model is given in Fig. 1. This system is slightly different from the existing ones in the following respects. A) Different from the existing studies on FD-NOMA, no relaying systems are used because of the vehicle's limited energy. B) V2X devices can directly communicate with each other through DC mode without the cellular's help, and the required contents are obtained from neighboring $\mathrm{V} 2 \mathrm{X}$ caches [33]. This system model thus has shorter transmission distance and better latency performance [33]. The cellular network load can be reduced too.

Once can notice that to simplify the analysis, only V2V and V2I communications are considered in the existing V2X studies, see, e.g., [25]-[27], [34]-[36]. As discussed, not only the vehicles, V2X aims to connect everything on the road. In order to cope with this trend, in our FD-NOMA-based decentralized V2X systems, all V2X devices (vehicle, pedestrian, traffic lights, etc.) are comprehensively included. The massive connected devices and their various applications are making the $\mathrm{V} 2 \mathrm{X}$ communications more complicated. To deal with this intractable problem, in this work, we classify the V2X communications into two scenarios: 1 ) the urban and crowded scenario and 2) the suburban and remote scenario.

In urban and crowded scenario, Rayleigh fading can be used as the channel model. This is due to the abundant reflection and refraction links between source and destination [37]. In contrast, Rician channel model is suitable for the suburban and remote scenario because of the less obstacles, where we can always establish a dominant light of sight (LoS) path from source to destination [38].

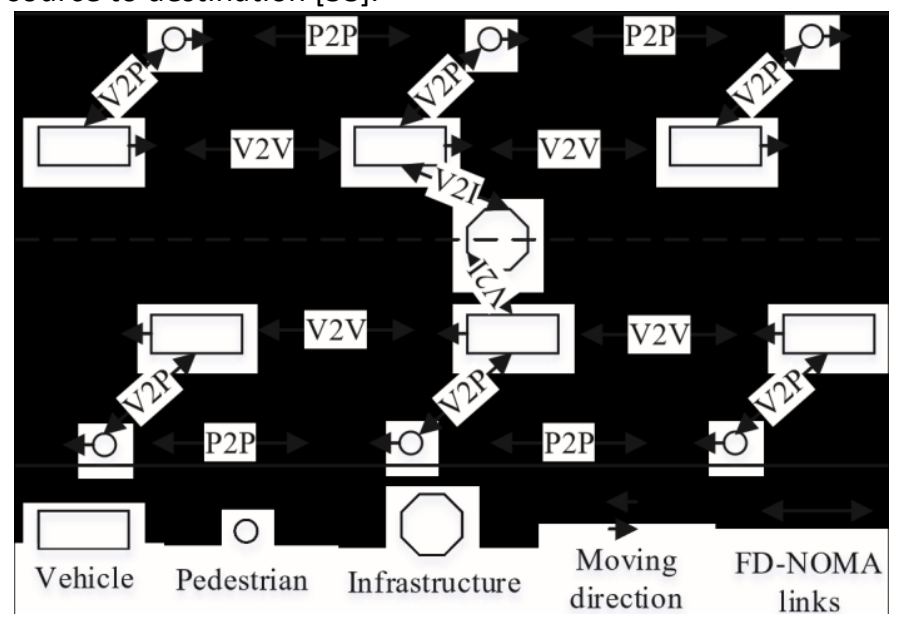

Fig. 1: The $M \leftrightarrow N$ FD-NOMA-based decentralized V2X system model. The communications among $V 2 X$ devices can be accomplished by FD-NOMA working on the DC mode.

\section{B. Received Signal and Power Allocation Scheme}

In the FD-NOMA-based decentralized V2X systems, the channel matrix from $M$ sources to $N$ destinations is

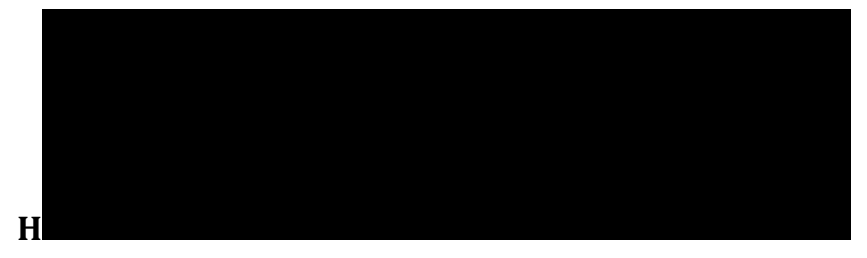

where $h_{i, j}$ is the channel between source $i$ and destination $j$. In this case, the received signal can be given as

$$
\mathbf{y}=\mathbf{H} \mathbf{p} \cdot \mathbf{x}+\mathbf{n},
$$

where $\mathbf{p} \bar{\in} \mathrm{C}$

$\sqrt{M \times 1}$, is the allocated downlink NOMA power 
matrix, $\mathbf{x} \in \mathrm{C}^{M \times 1}$ is the downlink transmit signal and $\mathbf{n} \sim$ $\mathrm{CN}\left(0, \sigma^{2} \mathbf{I}_{N}\right)$ is the downlink channel noise. Under the condition that $\hat{\mathbf{H}}=\mathbf{H}^{T}$ is the uplink channel with FD mode, uplink transmit information with FD mode will be

$$
\hat{\mathbf{y}}=\hat{\mathbf{H}} \hat{\mathrm{p}} \hat{\mathbf{p}} \cdot \mathbf{z}+\hat{\mathbf{n}},
$$

where $\mathbf{z} \in \mathrm{C}^{N \times 1}$ is the uplink information. NOMA power and channel noise vectors thus can be given as $\mathbf{p}^{\wedge}$. The total power received by destination $n$ from all $M$ sources is given by

$$
p_{n}=p 1, n+p 2, n+\ldots+p M, n
$$

Similarly,

$$
\hat{p}_{n}=\hat{p}_{n, 1}+\hat{p}_{n, 2}+\ldots+\hat{p}_{n, M},
$$

is the self-interference power when transmitting information to $M$ destinations from source $n$.

Remark 1: The received signal is composed of the received downlink information and its self-interference from the FD uplink. (1.10) On the other hand, transmission and reception processes in the FD-NOMA-based decentralized V2X systems are different from the centralized cellular-based communications, i.e., each $\mathrm{V} 2 \mathrm{X}$ destination can receive information with different NOMA power vectors from multiple distributed sources. By invoking the FD-NOMA techniques for simultaneous transmission and reception, the power received and transmitted by each V2X device are $p_{n}, \hat{p}_{n}$.

\section{ERgodic Capacity Analysis in Different SCEnarios}

In literature, capacity analysis is to reveal the intuitive and simple-to-compute capacity expressions for the wireless systems [39], [40]. In this regard, closed-form capacity expression is of great importance. Generally, capacity can be classified into two different types, i.e., the ergodic (Shannon) capacity and the outage capacity [41]. In time varying channels, on condition that the channel state information (CSI) is known at the receiver but not the transmitter, i.e., $\gamma$ (signal to interference plus noise ratio (SINR)) is known for every time slot. On condition that the CSI cannot be perfectly obtained because of the feedback delay or channel estimation error [42][44], the system performance is reduced. The hardware impairment (HI) is another issue that reduce the system performance. The impacts of imperfect CSI and $\mathrm{HI}$ to the NOMA-based systems have been investigated in prior works, see, e.g., [45], [46]. In literature, there are some methods to alleviate the side-effects of imperfect CSI and $\mathrm{HI}$, for instance, the deep learning-based CSI prediction [47]. In addition, some transmission methods without CSI have been investigated, see, e.g., [48].

On condition that the distribution of $\gamma$ is known at both the transmitter and receiver. Ergodic capacity is defined by data transmission going through all fading states, which is also called the Shannon capacity since it is the average of instantaneous capacity over all states. In contrast, outage capacity is used to describe the system performance under slowly varying channels with a constant instantaneous $\gamma$ [41], [49]. Here in this study, we adopt the ergodic capacity since V2X channels are generally the time varying channels.

In the decentralized FD-NOMA V2X systems, transmission channels are uncorrelated. In this case, the considered multiple input multiple output NOMA (MIMO-NOMA) can be treated as a sum of additive single input single output NOMA (SISONOMA) links. Moreover, similar to prior works [13], [50], we adopt an increasing order of the channel response, which means $\left|h_{i, 1}\right|^{2}$ $\leq, \ldots,\left|h_{i, j}\right|^{2} \leq, \ldots, \leq\left|h_{i, N}\right|^{2}, \forall i \in[1, M], j \in[1, N]$, vice versa. In this case, after successive interference cancellation (SIC), NOMA cochannel interference of the $i$-th user are from the $(i+1)$-th user to the $N$-th user [50].

According to Shannon theory [19], achievable capacity of each destination can be given by (6), see the equation in the top of next page. Here yields the co-channel interference from neighboring users after $\mathrm{SIC}, \eta \hat{p}_{i, k}$ is the selfinterference by FD uplink, $\sigma^{2}$ is the channel noise power, respectively. Additionally, $\eta$ is the coefficient of selfinterference with $\eta \in[0,1]$, which makes our expressions versatile to describe different schemes. For instance, in FDNOMA scheme, large value of $\eta$ denotes the strong FD selfinterference, and small value denotes the weak FD selfinterference. On condition that $\eta=0$, the expression reduces to the pure NOMA expression. On the basis of (6), normalizing the channel noise power value will give (7). Here $\rho$ is the SNR, and we use $\alpha_{i, j}, \alpha_{i, l}, \alpha_{i, k}$ to denote the allocated NOMA power coefficient with FD transmission in line with a normalized channel noise power value. In the sequel, we adopt the normalized noise power.

\section{A. Ergodic Capacity Analysis in Urban and Crowded Scenario}

We first analyze the achievable sum capacity in urban and crowded scenario. Note that we use the superscript $a$ and $c$ to distinguish different scenarios. In urban and crowded scenario, PDF of instantaneous signal to interference plus noise ratio (SINR) in each time slot, say, $\gamma_{i, j}$, is given by

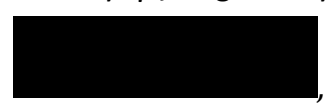

where

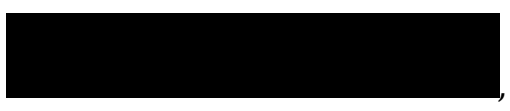

is the averaged channel power gain of each destination. As is well known, ergodic capacity is achieved by experiencing all the channel fading states, which means 


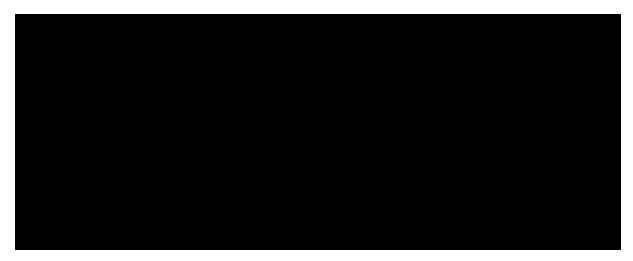

(10)

In the following theorem, we provide the exact ergodic capacity expression of the FD-NOMA-based decentralized V2X systems.

Theorem 1: In urban and crowded scenario, the exact achievable sum ergodic capacity of the FD-NOMA-based decentralized V2X systems is



where $E_{1}(x)$ is the exponential integral function that defined as

Additionally, we

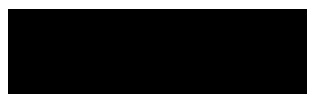

(9).

Proof: See Appendix A.

Exact ergodic capacity expression in urban and crowded scenario is provided in Theorem 1. Since the exponential integral function is involved, this expression thus is not given in closed-form. We thereby further pursue an approximate closedform expression of the achievable capacity. As noticed, in (11), the only expression not given by closed-form is the generalized exponential integral functions. In this case, our main focus is to find out a closed-form expression of $E_{1}(x)$.

$$
b_{k}=
$$$$
\theta k-1
$$

In addition, $\theta_{k}, k \in[0, n+1]$ is given by $0 \leq \theta_{0}<\theta_{1}<$ Besides, $a_{s}, b_{s}, \theta_{s}$ are defined with the same method, i.e.,

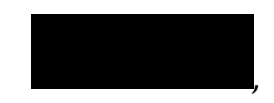

$$
b_{s}=\frac{\cot \theta_{s-1}-\cot \theta_{s}}{\theta_{s-1}}
$$

and

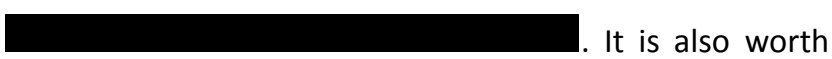

noting that the approximation accuracy is controlled by the division of with $\theta_{k}$ and $\theta_{s}$ (associate with $\left.a_{s}, b_{s}\right)^{2}$.

Proof: See Appendix B.

In order to verify the tightness of this expression, we compare the performances of the exact expression, the approximate expression and the well known Swamee and Ohija approximation. Note that the Swamee and Ohija approximation expression is given by [31]

where

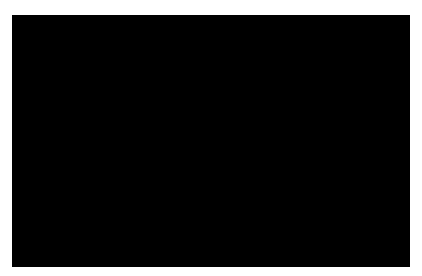

\section{(19)}

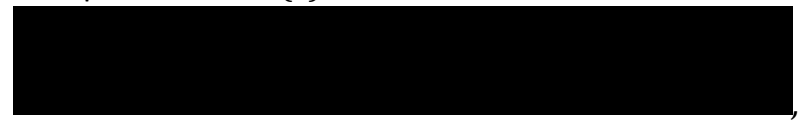

(6)

(7)
Lemma 1: Closed-form expression (lower bound) of the generalized exponential integral function is given by

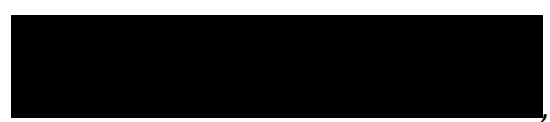

where $a_{k}, b_{k}$ are defined as

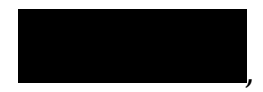

$$
\cot \theta_{k-1}-\cot \theta_{k}
$$

divide the with 1000 segments, which means, $\theta_{k}-\theta_{k-1}=$

. The simulation results are given by Fig. 2 . As noticed, the gap between the approximation and the exact form curves is large. Although this approximation method is better than the Swamee and Ohija approximation method, it is still unsuitable to be adopted directly.

We notice from Appendix B that in our derivations, the only issue that might bring in difference is the Jensen's inequality, i.e., in the derivations of $Q(x)$-function's closed-form expression, we use

\footnotetext{
${ }^{2}$ It is worth noting that here in our analysis, the equal division of $\mathbf{~ i s ~ u s e d . ~}$
} 


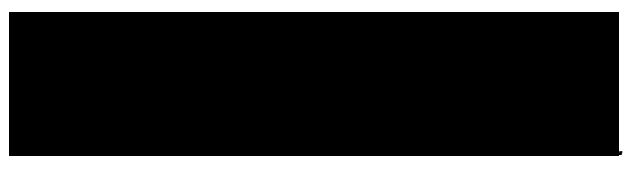

Additionally, one can see from Fig. 2 that the approximation curve displays a similar curvature to the exact curve. We can expect that a coefficient factor to the closed-form expression might improve the accuracy, i.e.,

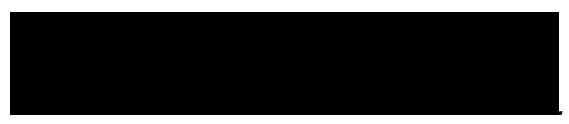

Consequently, our task is to find out a $q$ satisfying

Here we use

After some manipulations, we notice that when the above condition is met (e.g.,

$\left.E_{1}(1)|=| 0.2193827-0.2193839 \mid=1.2187 \times 10^{-6}\right)$. We thus have an approximate closed-form expression of $E_{1}(x)$ as

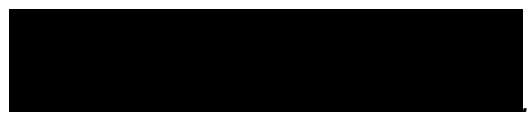

We further give the comparison results of the exact, improved and approximate expressions, which is shown in Fig. 3. Compared to the approximate results, the improved approximate results coincide with the exact results perfectly, which indicates the validity of our hypothesis. Closed-form expression of $C_{\text {sum }}{ }^{R a y}$ is given by the following corollary.

Corollary 1: By substituting (24) into (11), we obtain the approximate closed-form expression of the achievable capacity in urban and crowded scenario

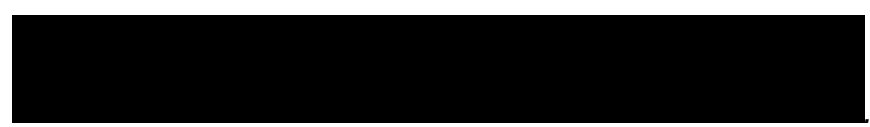

(25)

Remark 2: Insights from Corollary 1 is that the system ergodic capacity in urban and crowded scenario is determined by $M, N$, $\bar{\gamma}_{i, j}$. The system capacity increases with $M, N$. The accuracy of this approximate closed-form expression is determined by $n, t$. That is, the divisions of $\mathbf{l}$. The validity of this approximate expression will be verified by the following numerical results.

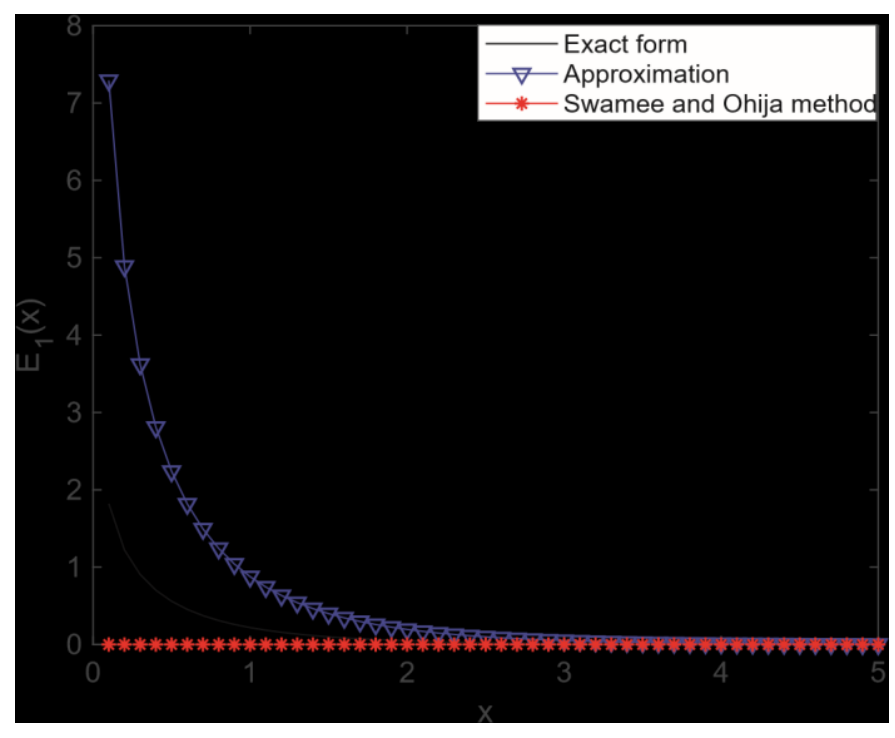

Fig. 2: Comparison of the exact, approximation and Swamee and Ohija-based expressions.

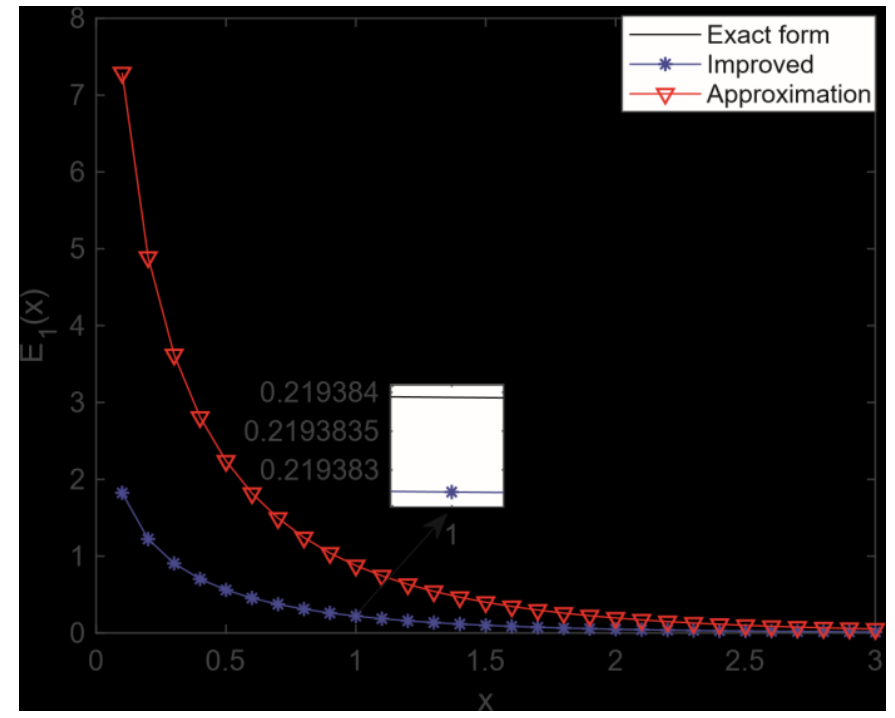

Fig. 3: Comparison of the exact, improved and approximate expressions.

\section{B. Ergodic Capacity Analysis in Suburban and Remote Scenario}

In the subsection III. A, we have obtained both exact and approximate forms of the capacity of the FD-NOMA-based decentralized V2X systems in urban and crowded scenario. In this subsection, we focus on the system capacity analysis in the suburban and remote scenario. We use $K$ as the Rician factor (which is the ratio between the deterministic and random fastfading component). It is noticed that in Rician channel, we have

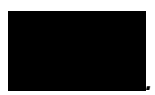


where $r^{2}$ yields the channel gain of LoS component, $2 \omega^{2}$ is the average channel power gain of all NLoS components. By defining the total average power gain as $\gamma^{-}$and following the prior work in [51], PDF of $\gamma_{i, j}$ can be given as



Here $I_{0}(\cdot)$ is the first kind modified Bessel function with zeroth order. By following a similar procedure of the previous analysis, we can obtain Theorem 2.

Theorem 2: Exact ergodic capacity expression of the FDNOMA-based decentralized V2X systems in suburban and remote scenario is given by

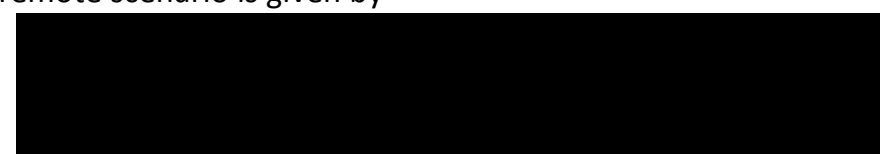

Here $E_{n}(x)$ is the generalized exponential integral function defined as [52]

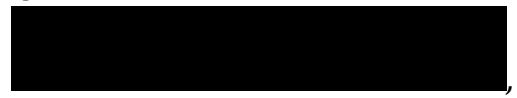

where $\operatorname{Re}(x)$ yields the real part of $x$.

Proof: See Appendix C.

This expression is still intractable to use directly because of the involved infinite factorial and generalized exponential integral expressions. In order to tame this troublesome problem, we give one approximate expression with arbitrary small error by invoking the truncation method in the sequel.

We find that the following expression

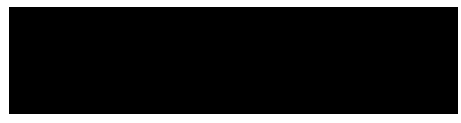

has an upper ceiling approximation, as shown by Corollary 2. In this case, the system capacity can be given by an approximate expression with much lower computation complexity and arbitrary small error, .

Corollary 2: By truncating the infinite series with regard to $T$, the capacity expression is approximately given as

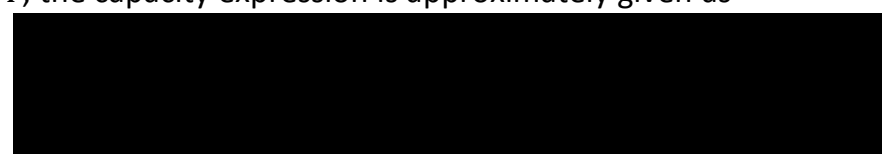

The truncation error is

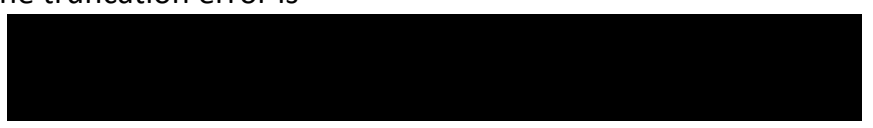

Proof: See Appendix D.

Remark 3: One can notice that the accuracy of the approximate expression in (31) is controlled by $T$. In other words, we may obtain an approximate expression with an arbitrary small error when



Insight from Corollary 2 has that the system capacity expression is determined by $M, N, \bar{\gamma}_{i, j}$ and $K$. With $M, N$ increasing, the system capacity always increases. The precise effects of $\bar{\gamma}_{i, j}, K$ to the capacity are still nonintuitive, which will be discussed in the following section.

\section{NUMERICAL RESULTS}

In this section, we perform the Monte Carlo simulations to verify the validity of our analysis. We also perform simulations to exposit the effects of different parameters to the system capacity, and compare the performance between FD-NOMA and NOMA schemes based on the decentralized FDNOMAenabled V2X systems. Due to variable parameters, we separately explain them and their values in the following simulations.

We first check the validity of the derived capacity expressions in (25) and (31). In these simulations, for the sake of compactness, one source with multiple destinations are used, where the source employs the FD-NOMA scheme to serve these destinations. We also assume that the allocated NOMA power variance is growing linearly with a normalized noise variance value (e.g., with 4 users, the NOMA power vector is $\mathbf{a}_{i}=$ $[4,3,2,1])$, where $\left.\mathbf{a}_{i}=\left[\alpha_{i, 1}, \ldots, \alpha_{i, N}\right]\right)$. Additionally, $\eta=0.1, \alpha_{i, k}=5$ are used. As clearly shown by Fig. 4 and

Fig. 5, our analytical results ${ }^{3}$ and the $M C$ results almost exactly coincide, which demonstrates the validity of our analysis. For instance, in Fig. 4, with $\rho=15 \mathrm{~dB}, 1 \leftrightarrow 4$, the $\mathrm{MC}$ and

App results are respectively 3.6865,3.6866 Bit/S/Hz. Under the same condition, as shown in Fig. 5, MC result and App result are 3.8458,3.8455 $\mathrm{Bit} / \mathrm{S} / \mathrm{Hz}$, respectively. The differences are less than $0.001 \mathrm{Bit} / \mathrm{S} / \mathrm{Hz}$ in both scenarios. We also observe that as the values of $N, \rho$ increases, the system capacity always increases. By comparing Fig. 4 and Fig. 5, we notice that under the same condition, capacities in suburban and remote scenario always outperform the ones in urban and crowded scenario (for instance, in $1 \leftrightarrow 3$ case, SNR = $0 \mathrm{~dB}$, ; SNR $=30 \mathrm{~dB}$, This is because of the less propagation loss with a dominant LoS path between source and destination in the suburban and remote scenario.

In order to verify the benefits of our analytical expressions, we compare the consumed time of App and MC simulations in Table I with $\rho=15 \mathrm{~dB}$ as an example. In these simulations, eight-core $3.4 \mathrm{GHz}$ processors, $16 \mathrm{~GB}$ memory and windows 10 64-bit operating system are used. The results are rounded off

\footnotetext{
${ }^{3}$ App: approximate, MC: Monte Carlo.
} 
to four decimal places. As shown in Table I, the consumed time of our analytical expressions are about $10^{6}$ times shorter than the MC simulations.

In the next step, we check the effect of Rician factor $K$ to the system capacity in suburban and remote scenario. In order to keep $K$ as the only variable, we do some manipulations as follows: 1) we keep all variables consistent except $K$; 2) with normalized noise power value and 3 destinations, we set $\mathbf{a}_{i}=$ $[1,2,3]$. The simulation results of the system capacity vs the destination number in suburban and remote scenario is given in Fig. 6. We notice that as the $K$ increases, system capacity



Fig. 4: Comparison of the system achievable sum capacity performances of App and MC results in urban and crowded scenario. The approximate results are obtained according to (25).



Fig. 5: Comparison of the system achievable sum capacity performances with App and $\mathrm{MC}$ results in suburban and remote scenario. The analytical results are obtained according to (31). also increases. This is because the higher $K$ brings in a stronger LoS component and a weaker multi-path propagation loss.

Besides the effects of $N, \rho, K$, the effects of $M$ and $\mathbf{a}_{i}$ to the system capacity are also checked with: 1) a linearly growing power value with $M=1$ (i.e., $\mathbf{a}_{1}=[0.5,1,1.5], \mathbf{a}_{2}=[1,2,3], \mathbf{a}_{3}=$ $[2,4,6]) ; 2$ ) different NOMA power vectors with $M=2$ (i.e., $2 \leftrightarrow$ $3, \mathbf{a}_{1}, \mathbf{a}_{3}$ denote that two sources are transmitting information to 3 destinations with FD-NOMA, where the NOMA power vector are $\mathbf{a}_{1}, \mathbf{a}_{2}$, respectively). The simulation results are given by Fig. 7 and Fig. 8. As shown by the solid lines in both figures, increasing the power values leads 
TABLE I: Consumed time (second) of App and MC simulations with $\rho=15 \mathrm{~dB}$.



Fig. 6: Comparison of the capacities with different power values and source numbers.

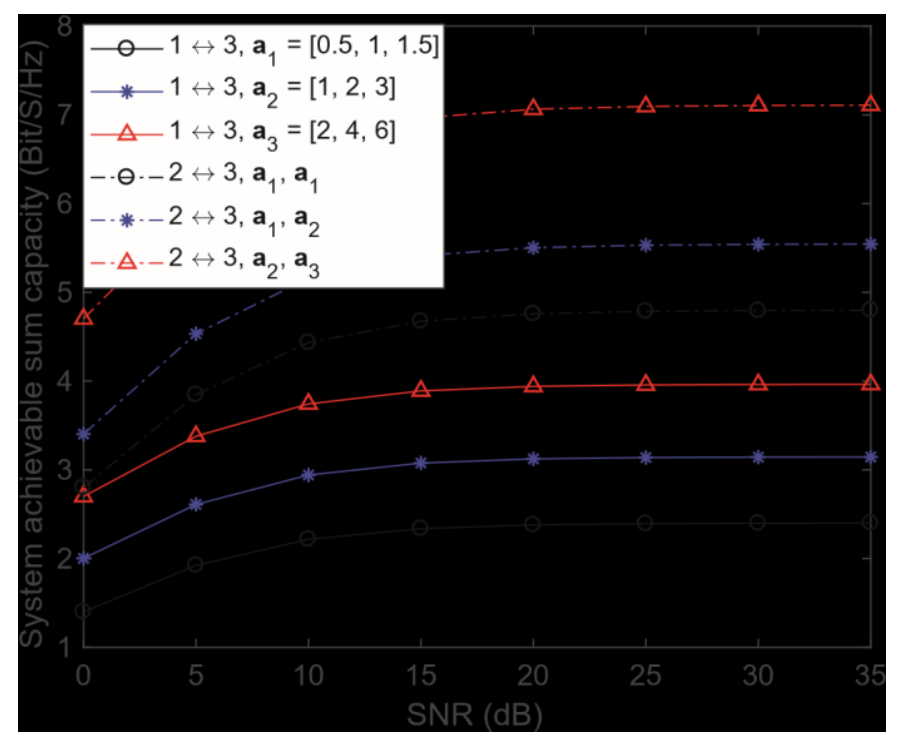

Fig. 7: Comparison of the capacities with different power values and source numbers.

to better capacity performance, which is due to the increased SNR value. For instance, in $1 \leftrightarrow 3$ case and SNR $=20 \mathrm{~dB}$, we have We can also confirm from both figures that as $M$ increases, the system capacities also increase.

Finally, we compare the achievable throughputs with FD-
NOMA, NOMA, FD-OMA and HD-OMA schemes in different Fig. 8: Comparison of the capacities with different power values and source numbers.

scenarios. The results are given in Fig. 9 and Fig. 10. In these simulations, carrier bandwidth $B=100 \mathrm{MHz}, \mathbf{a}_{i}=[3,2,1], \eta=0.1$ and $\alpha_{i, k}=0.1,1,10$ are used. In order to be fair, we average the allocated power in FD-OMA and HD-OMA schemes. As shown in both figures, NOMA scheme has a better throughput performance compared to OMA scheme. Moreover, with a smaller value of $\alpha_{i, k}$, FD-NOMA always outperforms the other schemes (HD-NOMA, FD-OMA, HD-OMA). However, the benefit of FD-NOMA decreases while $\alpha_{i, k}$ increasing. This is mainly due to the increased FD self-interferences. We also notice that even with a higher FD self-interference value, FD-NOMA outperforms NOMA in low SNR scenario (i.e., $\rho \in[0,5] \mathrm{dB}$ ). This is because in low SNR scenario, channel noise is the dominant factor compared to FD self-interference. In contrast, FD-NOMA self-interference becomes the dominant factor in high SNR scenario, NOMA scheme without FD selfinterference thus has a better throughput performance. It is also worth noting that the effective transmission time is limited because of the fast moving V2X devices. FD-NOMA enabled bidirectional transmission can greatly reduce the transmission latency compared to other schemes. For example, compared to HDNOMA and HD-OMA, FD-NOMA only needs a half latency time to transmit the same amount of data by its simultaneous transmission and reception scheme. 


\section{CONCLUSION}

In this article, we proposed the FD-NOMA-based decentralized V2X systems. We classified the V2X communications

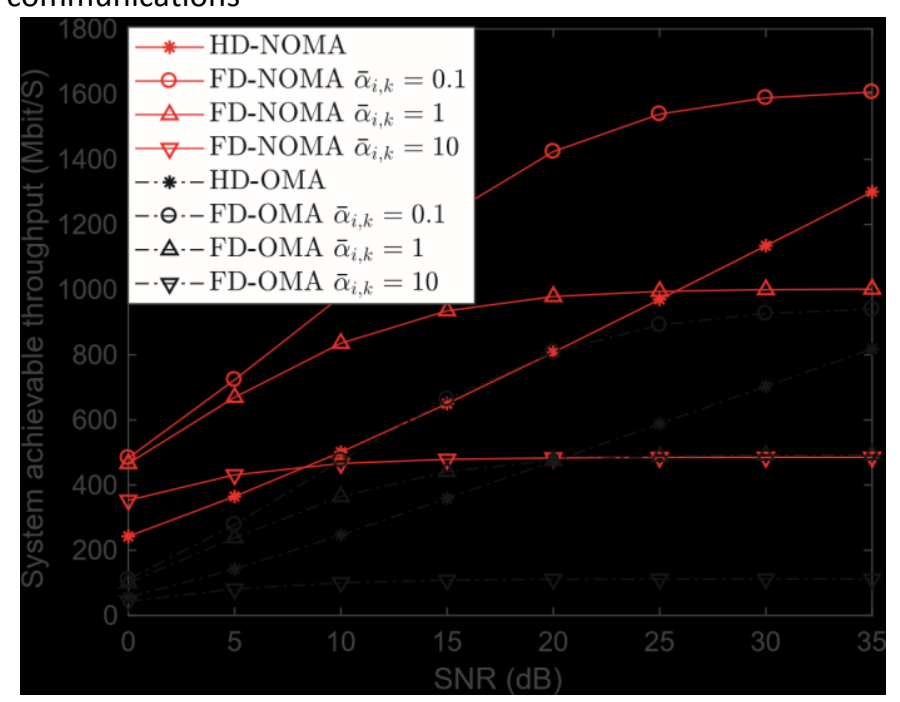

Fig. 9: System achievable throughput comparisons with FDNOMA, NOMA, FD-OMA and HD-OMA schemes in urban and crowded scenario.

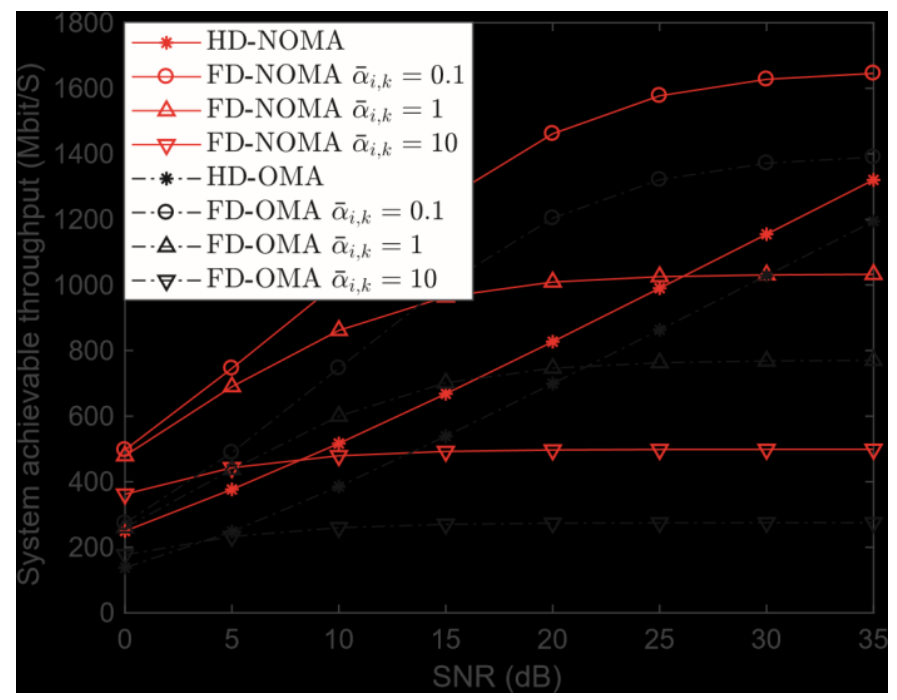

Fig. 10: System achievable throughput comparisons with FDNOMA, NOMA, FD-OMA and HD-OMA schemes in suburban and remote scenario.

into two typical scenarios, i.e., the urban and crowd scenario and the suburban and remote scenario, and then derived the exact system capacity expressions in both scenarios. To tackle down the capacity expression's intractable calculations in both scenarios, we further obtained their simplified approximate expressions. Insights of our analysis are that the accuracy of our simplified approximate expression in urban and crowded scenario is determined by the associated division of $\mathbf{D i t h}$ respect to exponential integral function $\left(E_{1}(x)\right)$, and the accuracy of simplified approximate expression in suburban and remote scenario is determined by the truncation point $T$ (with respect to generalized exponential integral function $\left(E_{n}(x)\right)$. Numerical results demonstrate the validity and effectiveness of our analytical results. Compared to MC method, the consumed time is greatly reduced by our Approximation expressions. Simulation results also demonstrated that the system capacity performance can be enhanced by increasing the number of V2X devices, NOMA power and Rician factor (suburban and remote scenario), and the effectiveness of FD-NOMA is determined by the FD self-interference and the channel noise. In addition, FDNOMA can greatly reduce the system latency compared to other schemes.

\section{APPENDIX A: ProOf OF THEOREM 1}

Firstly, according to the integration by parts method, we have

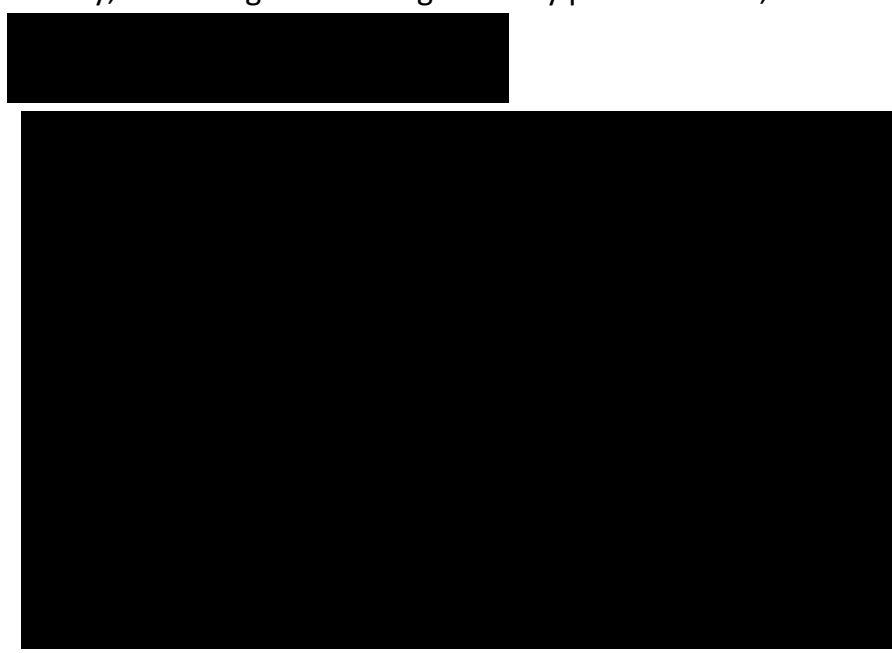

So far the expression is still intractable. In the next step, we recall the alternative generalized exponential integral expression

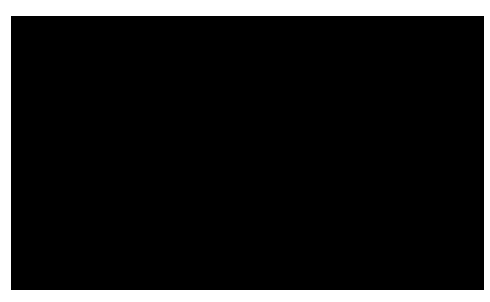

[52]

By substituting (A.2) into (A.1), and further summarizing the result with $M$ sources and $N$ destinations, we can safely arrive the final expression.

This completes the proof.

ApPendix B: ProOf OF LeMma 1

As noticed, $E_{1}(x)$ can be rewritten as 




It is also noticed that the following equality holds [53]

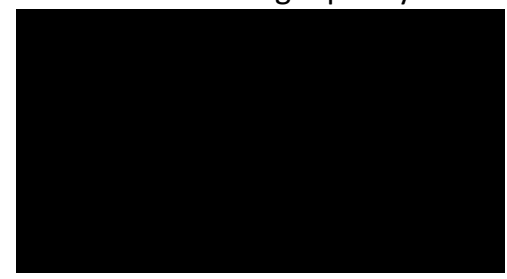

The approximate accuracy of this lower bound expression is controlled by the interval gap between each pair of $\left[\theta_{k-1}, \theta_{k}\right]$. Moreover, it is known that the following equality holds

$\mathrm{Z}$

$$
\sin ^{-2} x d x=-\cot x+C
$$

Substituting it into (B.6), we thus have

Thus in the next step, our work is to seek a closed-form expression for the $Q$-function. Actually, there are various closedform expressions to capture the lower or upper bounds of the $Q$-function, for instance, the chernoff bound

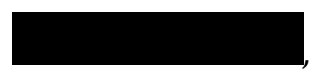

the improved exponential bound



However, the integral is still intractable while substituting those expressions into (B.1), an alternative method is needed. According to prior work, by adopting the Craig's form, we have [53]

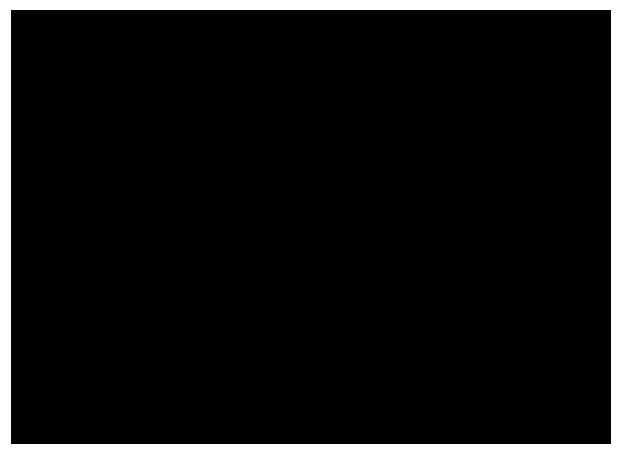

with $a_{k}, b_{k}$ are defined as

$\theta \theta_{k k-1}-1 \theta-k-\cot 1 \theta$.

Then by substituting the Jensen's inequality [54] to (B.5), we have the lower bound expression of $Q$-function as

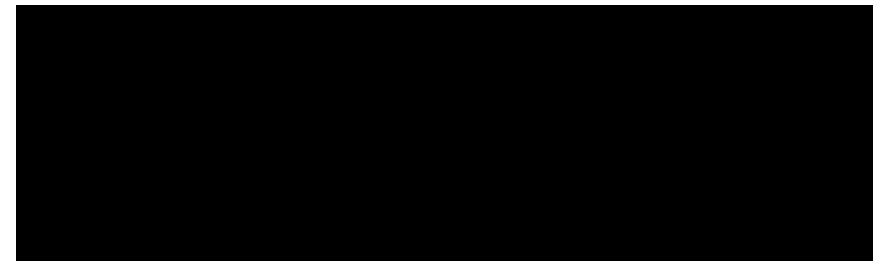

Additionally, it is worth noting that $\theta_{k}, k \in[0, n+1]$ is given by



Finally, by substituting (B.2) into (B.1), $E_{1}(x)$ can be given as

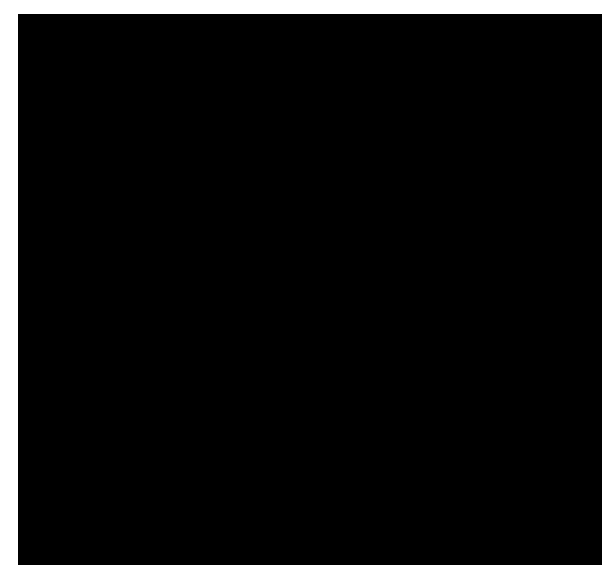

As the final inequality is obtained while substituting the ap $-\sqrt{ }$ proximate expression of $Q\left(2 b_{k} x\right)$, definitions of $a_{s}, b_{s}, \theta_{s}$ thus are similar as prior definitions of $a_{k}, b_{k}, \theta_{k}$, i.e., $a_{s}=$

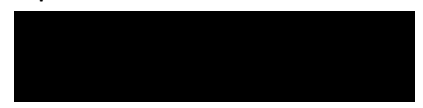

and $0 \leq \theta_{0}<\theta_{1}<\ldots \theta_{s}<\ldots<$

This completes the proof.

APPENDIX C: PROOF OF THEOREM 2

It is noticed that the PDF of $\gamma_{i, j}$ in Rician channel condition can be given by [51] 
By following a similar derivation procedure as in Theorem 1, we have the following equation

$$
\begin{aligned}
& C_{i, j c}=\mathrm{E}\left[\log _{2}\left(1+{ }^{-} \gamma_{i, j}\right)\right] \\
& =\begin{array}{cc} 
& \mathrm{Z}^{+\infty} \quad \text { (C.2) } c \\
& \quad \log _{2}\left(1+\gamma_{i, j}\right) f\left(\gamma_{i, j}\right) d \gamma_{i, j .} .
\end{array}
\end{aligned}
$$

and substituting equations (C.4) and (C.5) into this expression, the derivations of capacity expression of the FD-NOMA-based decentralized $\mathrm{V} 2 \mathrm{X}$ systems can be given as:

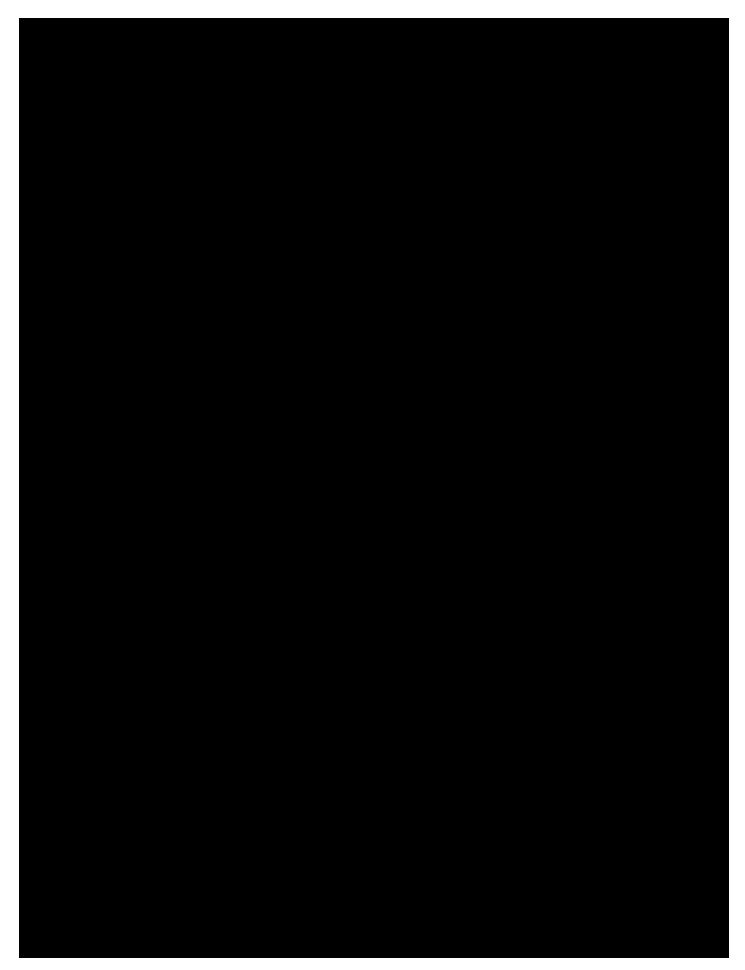

Here the second equality is due to the modified Bessel function of the zeroth order expression [55]



Additionally, the third equality is because [55]

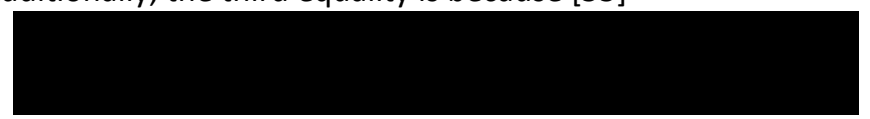

By further summarizing this expression with $\mathrm{M}$ sources and $\mathrm{N}$ destinations, we can finally arrive at (31). This completes the proof.

\section{Appendix D: Proof of Corollary 2}

The remaining section after a truncation with regard to $T$ is

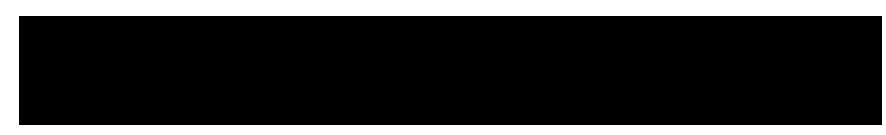

As shown here, approximate error mainly comes from the infinite expression series with regard to $m$. According to prior work in [56], [57], $E_{n}(x)$ monotonically decreasing in $n$ giving equal $x$. In this case, by putting

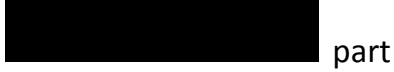
aside, we have the following expression

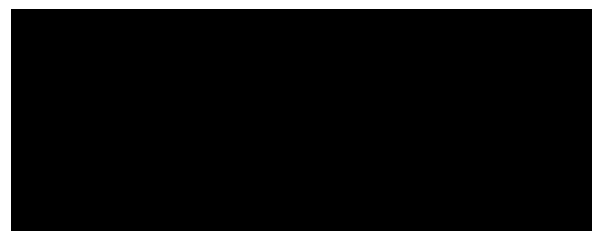

It is noticed that giving constant values of $\bar{\gamma}_{i, j}$ and then becomes a constant coefficient. Consequently, we focus on the function

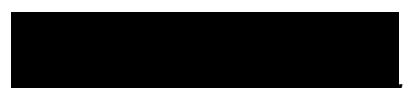

By some mathematical manipulations, it is found that there existing $x^{0}$, so that $f^{0}\left(x^{0}\right)=0$ with $f^{00}\left(x^{0}-\right)>0, f^{00}\left(x^{0}+\right)<0$. Additionally, observation has that $f(x)$ rapidly converges to 0 after $x^{0}$ (e.g., $\left.f(100)=6.9966 \times 10^{-125}\right)$. This gives approximate capacity expression of (31) with an arbitrary small error .

This completes the proof.

\section{REFERENCES}

[1] Z. Zhou, H. Yu, C. Xu, Y. Zhang, S. Mumtaz, and J. Rodriguez, "Dependable content distribution in D2D-based cooperative vehicular networks: A big data-integrated coalition game approach," IEEE Trans. Intell. Transp. Syst., vol. 19, no. 3, pp. 953-964, Mar. 2018.

[2] J. B. Kenney, "Dedicated short-range communications (DSRC) standards in the united states," Proc. IEEE, vol. 99, no. 7, pp. 1162-1182, Jul. 2011.

[3] B. Walke, W. Mende, and G. Hatziliadis, "CELLPAC: A packet radio protocol applied to the cellular GSM mobile radio network," in IEEE VTC, May 1991, pp. 408-413.

[4] W. Zhu, T. Hellmich, and B. Walke, "Dcap, a decentral channel access protocol: performance analysis," in IEEE VTC, May 1991, pp. 463-468.

[5] Z. Zhu, Z. Wang, Z. Chu, D. Zhang, and B. Shim, "Robust energy harvest balancing optimization with V2X-SWIPT over MISO secrecy channel," Comput. Netw., vol. 137, no. 4, pp. 61-68, Jun. 2018.

[6] Z. Xu, X. Li, X. Zhao, M. H. Zhang, and Z. Wang, "DSRC versus 4GLTE for connected vehicle applications: A study on field experiments of vehicular communication performance," J. Adv. Transp., vol. 2017, pp. 1-10, Aug. 2017.

[7] G. Americas, "Cellular V2X communications towards 5G," $5 \mathrm{G}$ Americas, Tech. Rep., Mar. 2018.

[8] L. Liang, H. Peng, G. Y. Li, and X. Shen, "Vehicular communications: A physical layer perspective," IEEE Trans. Veh. Tech., vol. 66, no. 12, pp. 10647-10659, Dec. 2017.

[9] B. Hui, J. Kim, H. Chung, I. Kim, and H. Lee, "Efficient doppler mitigation for high-speed rail communications," in IEEE ICACT, Jan. 2016, pp. 634638.

[10] A. Gomaa and L. M. A. Jalloul, "Receiver architecture for frequency offset correction and $\mathrm{I} / \mathrm{Q}$ imbalance compensation in equal bandwidth contiguous carrier aggregation," IEEE Trans. Veh. Technol., vol. 65, no. 10, pp. 8730-8735, Oct. 2016. 
[11] H. Ji, S. Park, J. Yeo, Y. Kim, J. Lee, and B. Shim, "Ultra-reliable and lowlatency communications in $5 \mathrm{G}$ downlink: Physical layer aspects," IEEE Wireless Commun., vol. 25, no. 3, pp. 124-130, Jun. 2018.

[12] J. Wu, M. Dong, K. Ota, J. Li, and Z. Guan, "Big data analysis-based secure cluster management for optimized control plane in softwaredefined networks," IEEE Trans. Netw. Service Manag., vol. 15, no. 1, pp. 27-38, Mar. 2018.

[13] X. Yue, Y. Liu, S. Kang, A. Nallanathan, and Z. Ding, "Exploiting full/halfduplex user relaying in NOMA systems," IEEE Trans. Commun., vol. 66, no. 2, pp. 560-575, Feb. 2018.

[14] W. Liang, Z. Ding, Y. Li, and L. Song, "User pairing for downlink nonorthogonal multiple access networks using matching algorithm," IEEE Trans. Commun., vol. 65, no. 12, pp. 5319-5332, Dec. 2017.

[15] D. Zhang, Y. Liu, Z. Ding, Z. Zhou, A. Nallanathan, and T. Sato, "Performance analysis of non-regenerative Massive-MIMO-NOMA relay systems for 5G," IEEE Trans. Commun., vol. 65, no. 11, pp. 4777-4790, Nov. 2017.

[16] L. Dai, B. Wang, Y. Yuan, S. Han, C. I. I, and Z. Wang, "Non-orthogonal multiple access for $5 \mathrm{G}$ : solutions, challenges, opportunities, and future research trends," IEEE Commun. Mag., vol. 53, no. 9, pp. 74-81, Sep. 2015.

[17] H. Zhang, N. Yang, K. Long, M. Pan, G. K. Karagiannidis, and V. C. M. Leung, "Secure communications in NOMA system: Subcarrier assignment and power allocation," IEEE J. Sel. Areas Commun., vol. 36, no. 7, pp. 14411452, Jul. 2018.

[18] H. Zhang, N. Yang, K. Long, M. Pan, G. K. Karagiannidis, and A. Nallanathan, "Energy efficient resource allocation for secure NOMA networks," in IEEE VTC Spring, Jun. 2018, pp. 1-6.

[19] Y. Liu, M. Elkashlan, Z. Ding, and G. K. Karagiannidis, "Fairness of user clustering in MIMO non-orthogonal multiple access systems," IEEE Commun. Lett., vol. 20, no. 7, pp. 1465-1468, Jul. 2016.

[20] F. Fang, H. Zhang, J. Cheng, and V. C. M. Leung, "Energy-efficient resource scheduling for NOMA systems with imperfect channel state information," in IEEE ICC, May 2017, pp. 1-5.

[21] A. Sabharwal, P. Schniter, D. Guo, D. W. Bliss, S. Rangarajan, and R. Wichman, "In-band full-duplex wireless: Challenges and opportunities," IEEE J. Sel. Areas Commun., vol. 32, no. 9, pp. 1637-1652, Sep. 2014.

[22] Z. Ding, P. Fan, and H. V. Poor, "On the coexistence between full-duplex and NOMA [early access:] https://ieeexplore.ieee.org/document/8306094/," IEEE Wireless Commun. Lett., Mar. 2018.

[23] H. Huang, J. Xiong, J. Yang, G. Gui, and H. Sari, "Rate region analysis in a full-duplex-aided cooperative nonorthogonal multiple-access system," IEEE Access, vol. 5, pp. 17869-17880, Aug. 2017.

[24] M. F. Kader, S. Y. Shin, and V. C. M. Leung, "Full-duplex non-orthogonal multiple access in cooperative relay sharing for $5 \mathrm{G}$ systems," IEEE Trans. Veh. Tech., vol. 67, no. 7, pp. 5831-5840, Jul. 2018.

[25] B. Di, L. Song, Y. Li, and G. Y. Li, "Non-orthogonal multiple access for highreliable and low-latency V2X communications in $5 \mathrm{G}$ systems," IEEE J. Sel. Areas Commun., vol. 35, no. 10, pp. 2383-2397, Oct. 2017.

[26] B. W. Khoueiry and M. R. Soleymani, "An efficient NOMA V2X communication scheme in the internet of vehicles," in IEEE VTC, Jun. 2017, pp. 1-7.

[27] M. Yang, S. W. Jeon, and D. K. Kim, "Interference management for inband full-duplex vehicular access networks," IEEE Trans. Veh. Technol., vol. 67, no. 2, pp. 1820-1824, Feb. 2018.

[28] M. D. Yacoub, "The $\kappa-\mu$ distribution and the $\eta-\mu$ distribution," IEEE Antennas and Propag. Mag., vol. 49, no. 1, pp. 68-81, Feb. 2007.

[29] N. Bhargav, S. L. Cotton, and D. E. Simmons, "Secrecy capacity analysis over $\kappa$ c $\mu$ fading channels: Theory and applications," IEEE Trans. Commun., vol. 64, no. 7, pp. 3011-3024, Jul. 2016.

[30] X. Li, J. Li, L. Li, J. Jin, J. Zhang, and D. Zhang, "Effective rate of miso systems over $\kappa-\mu$ shadowed fading channels," IEEE Access, vol. 5, pp. 1060510611, Jun. 2017.

[31] G. P. Huy, "Revisit of well function approximation and an easy graphical curve matching technique for theis solution," Groundwater, vol. 41, no. 3, pp. 387-390, Dec. 2005.
[32] G. Navas-Palencia, "Fast and accurate algorithm for the generalized exponential integral $e_{v}(\mathrm{x})$ for positive real order," Numerical Algorithms, vol. 77, no. 2, pp. 603-630, Feb. 2018.

[33] D. Zhang, Z. Zhou, S. Mumtaz, J. Rodriguez, and T. Sato, "One integrated energy efficiency proposal for 5G IOT communications," IEEE Internet Things J., vol. 3, no. 6, pp. 1346-1354, Dec. 2016.

[34] A. Bazzi, B. M. Masini, and A. Zanella, "How many vehicles in the LTE-V2V awareness range with half or full duplex radios?" in 2017 15th International Conference on ITS Telecommunications (ITST), May 2017, pp. 1-6.

[35] P. Wang, B. Di, H. Zhang, K. Bian, and L. Song, "Cellular V2X communications in unlicensed spectrum: Harmonious coexistence with VANET in 5G systems," IEEE Trans. Wireless Commun., 2018.

[36] M. Boban, K. Manolakis, M. Ibrahim, S. Bazzi, and W. Xu, "Design aspects for $5 \mathrm{G}$ V2X physical layer," in IEEE CSCN, Oct. 2016, pp. 1-7.

[37] R. J. C. Bultitude and G. K. Bedal, "Propagation characteristics on microcellular urban mobile radio channels at $910 \mathrm{MHz}$," IEEE J. Sel. Areas Commun., vol. 7, no. 1, pp. 31-39, Jan. 1989.

[38] Q. Zhang, T. Q. S. Quek, and S. Jin, "Scaling analysis for massive MIMO systems with hardware impairments in Rician fading [early access:] https://ieeexplore.ieee.org/document/8350341/," IEEE Trans. Wireless Commun., Apr. 2018.

[39] C. E. Shannon, "A mathematical theory of communication," Bell Labs Syst. Tech. J., vol. 27, no. 3, pp. 379-423, Jul. 1948.

[40] S. Weber, J. G. Andrews, and N. Jindal, "An overview of the transmission capacity of wireless networks," IEEE Trans. Commun., vol. 58, no. 12, pp. 3593-3604, Dec. 2010.

[41] S. Choudhury and J. D. Gibson, "Information transmission over fading channels," in IEEE GLOBECOM, Nov. 2007, pp. 3316-3321.

[42] H. Y. H. Q. N. Thomas L Marzetta, Erik G Larsson, "Fundamentals of massive mimo," Cambridge, UK, 2016.

[43] S. S. Wei Xiang, Kan Zheng, "5G mobile communications," AG, Switzerland, 2017.

[44] T. Van Chien, C. Molln, and E. Bjrnson, "Large-scale-fading decoding in cellular massive MIMO systems with spatially correlated channels [early access:] https://ieeexplore.ieee.org/document/8585131," IEEE Trans. Commun., 2018.

[45] P. T. M. D. Z. L. L. X. Li, J. Li and J. Jin, "Joint impact of hardware impairments and imperfect CSI on cooperative SWIPT NOMA multirelaying systems," in IEEE ICCC, Aug. 2018, pp. 95-99.

[46] D. Z. X. L. J. L. C. C. C. C. Deng, X. Zhao, "Performance analysis of nomabased relaying networks with transceiver hardware impairments," vol. 12, no. 9, pp. 4295-4316, Sep. 2018.

[47] C. Luo, J. Ji, Q. Wang, X. Chen, and P. Li, "Channel state information prediction for $5 \mathrm{G}$ wireless communications: A deep learning approach [early access:] https://ieeexplore.ieee.org/document/8395053," IEEE Trans. Netw. Sci. Eng., 2018.

[48] S. A. Jafar, "Blind interference alignment," IEEE J. Sel. Topics Signal Process., vol. 6, no. 3, pp. 216-227, Jun. 2012.

[49] A. Goldsmith, "Wireless communciations," New York, USA, 2005.

[50] Y. Liu, Z. Ding, M. Elkashlan, and H. V. Poor, "Cooperative nonorthogonal multiple access with simultaneous wireless information and power transfer," IEEE J. Sel. Areas Commun., vol. 34, no. 4, pp. 938-953, Apr. 2016.

[51] H. C. Yang and M. S. Alouini, "Order statistics in wireless communications: Diversity, adaptation, and scheduling in mimo and ofdm systems," New York, USA, 2011.

[52] I. S. Gradshteyn and I. M. Ryzhik, "Table of integrals, series and products," London, UK, 2015.

[53] A. A. Alkheir and M. Ibnkahla, "An accurate approximation of the exponential integral function using a sum of exponentials," IEEE Commun. Lett., vol. 17, no. 7, pp. 1364-1367, Jul. 2013.

[54] M. Wu, X. Lin, and P. Y. Kam, "New exponential lower bounds on the Gaussian Q-Function via Jensen's inequality," in IEEE VTC Spring, May 2011, pp. 1-5. 
[55] A. L. Giuseppa Alfano, A. M. Tulino, and S. Verdu, "Mutual information and eigenvalue distribution of MIMO Rician channels," in IEEE ISITA, Parma, Italy, Oct. 2004, pp. 1-6.

[56] J. Zhang, L. Dai, X. Zhang, E. Bjonson, and Z. Wang, "Achievable" rate of Rician large-scale MIMO channels with transceiver hardware impairments," IEEE Trans. Veh. Tech., vol. 65, no. 10, pp. 8800-8806, Oct. 2016.

[57] C. Chiccoli, S. Lorenzutta, and G. Maino, "Recent results for generalized exponential integrals," Comput. Math. Appl., vol. 19, no. 5, pp. $21-29$, 1990.

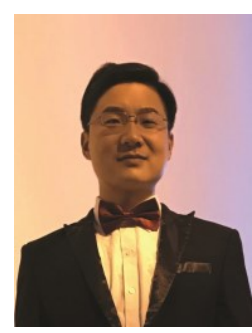

Di Zhang (S'13-M'17) is an Assistant Professor with the Zhengzhou University, Zhengzhou, China, and also a visiting researcher with the Seoul National University, Seoul, Korea. He received his Ph.D. degree from the Waseda University, Tokyo, Japan, in 2017. He visited the National Key Laboratory of

Alternate Electrical Power System with Renewable Energy Sources, Beijing, China, from 2015 to 2017, and the National Chung Hsing University, Taichung, Taiwan, in 2012. He serves as the editor of the KSII

TRANSACTIONS ON INTERNET AND INFORMATION SYSTEMS, has served as the guest editors of the IEEE NETWORK, the IEEE

ACCESS, the IET INTELLIGENT TRANSPORT SYSTEMS, TPC members of many IEEE flagship conferences, such as ICC and WCNC. His research interests include 5G wireless networks, internet of things and telemedicine.

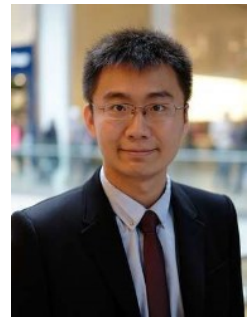

Yuanwei Liu (S'13-M'16) received the B.S. and M.S. degrees from the Beijing University of Posts and Telecommunications in 2011 and 2014, respectively, and the Ph.D. degree in electrical engineering from the Queen Mary University of London, U.K., in 2016. He was with the Department of Informatics, King's College London, from 2016 to 2017, where he was a PostDoctoral Research Fellow. He has been a Lecturer (Assistant Professor) with the School of Electronic Engineering and Computer Science, Queen Mary University of London, since 2017.

His research interests include $5 \mathrm{G}$ wireless networks, Internet of Things, machine learning, stochastic geometry, and matching theory. He received the Exemplary Reviewer Certificate of the IEEE WIRELESS COMMUNICATION LETTERS in 2015 and the IEEE TRANSACTIONS ON COMMUNICATIONS and the IEEE TRANSACTIONS ON WIRELESS COMmUNICATIONS in 2016 and 2017. He has served as a TPC Member for many IEEE conferences, such as GLOBECOM and ICC. He currently serves as an Editor of the

IEEE TRANSACTIONS ON COMMUNICATIONS, the IEEE COMMUNICATIONS

LETTERS and the IEEE ACCESS. He is also a guest editor for IEEE JSTSP special issue on "Signal Processing Advances for Non-Orthogonal Multiple Access in Next Generation Wireless Networks".

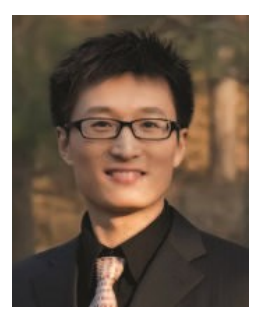

Linglong Dai (M'11-SM'14) received the B.S. degree from Zhejiang University in 2003, the M.S. degree (with the highest honor) from the China Academy of Telecommunications Technology in 2006, and the Ph.D. degree (with the highest honor) from Tsinghua University, Beijing, China, in 2011. From 2011 to 2013, he was a Post-Doctoral Research Fellow with the Department of Electronic Engineering, Tsinghua University, where he was an Assistant Professor from 2013 to 2016 and has been an Associate Professor since 2016. He co-authored the book " $m$ Wave Mas-

sive MIMO: A Paradigm for 5G" (Academic Press, Elsevier, 2016). He has published over 60 IEEE journal papers and over 40 IEEE conference papers. He also holds 16 granted patents. His current research interests include massive MIMO, millimeter-wave communications, NOMA, sparse signal processing, and machine learning for wireless communications. He has received five IEEE Best Paper Awards at the IEEE ICC 2013, the IEEE ICC 2014, the IEEE ICC 2017, the IEEE VTC 2017-Fall, and the IEEE ICC 2018. He has also received the Tsinghua University Outstanding Ph.D. Graduate Award in 2011, the Beijing Excellent Doctoral Dissertation Award in 2012, the China National Excellent Doctoral Dissertation Nomination Award in 2013, the URSI Young

Scientist Award in 2014, the IEEE Transactions on Broadcasting Best Paper

Award in 2015, the Electronics Letters Best Paper Award in 2016, the National Natural Science Foundation of China for Outstanding Young Scholars in 2017, the IEEE ComSoc Asia-Pacific Outstanding Young Researcher Award in 2017, and the IEEE ComSoc Asia-Pacific Outstanding Paper Award in 2018. He currently serves as an Editor of the IEEE Transactions on Communications, the IEEE Transactions on Vehicular Technology, and the IEEE Communications Letters. Particularly, he is dedicated to reproducible research and has made a large amount of simulation code publicly available.

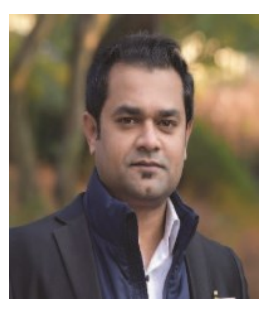

Ali Kashif Bashir (M'15-SM'16) is a Senior Lecturer at School of Computing, Mathematics, and Digital Technology, Manchester Metropolitan University, U.K. He is a senior member of IEEE and Distinguished Speaker of ACM. His past assignments include: Associate Professor of Information and Communication Technologies, Faculty of Science and Technology, University of the Faroe Islands, Denmark; Osaka University, Japan; Nara National College of Technology, Japan; the National Fusion Research Institute, South Korea; Southern Power Company Ltd., South Korea, and the Seoul Metropolitan Government, South Korea. He received his Ph.D. in computer science and engineering from Korea University, South Korea. MS from Ajou University, South Korea and BS from University of Management and Technology, Pakistan. He is author of over 80 peer-reviewed articles. He is supervising/co-supervising several graduate (MS and $\mathrm{PhD}$ ) students. His research interests include internet of things, wireless networks, distributed systems, network/cyber security, cloud/network function virtualization, etc. He is serving as the Editor-in-chief of the IEEE FUTURE DIRECTIONS NEWSLETTER.

He is editor of several journals and also has served/serving as guest editor on several special issues in journals of IEEE, Elsevier, and Springer. He has served as chair (program, publicity, and track) chair on several conferences and workshops. He has delivered several invited and keynote talks, and reviewed the technology leading articles for journals, such as the IEEE TRANSACTIONS ON INDUSTRIAL INFORMATICS, the IEEE COMMUNICATION MAGAZINE, the IEEE COMMUNICATIONS LETTERS, IEEE INTERNET OF THINGS, and the IEICE Journals, and conferences, such as the IEEE INFOCOM, the IEEE ICC, the IEEE GLOBECOM, and the IEEE Cloud of Things. 


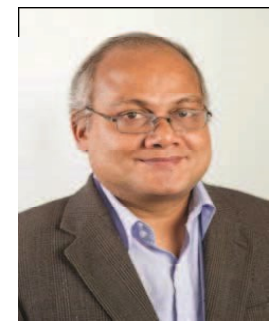

Arumugam Nallanathan (S'97-M'00-SM'05-F'17) is Professor of Wireless Communications and Head of the Communication Systems Research (CSR) group in the School of Electronic Engineering and Computer Science at Queen Mary University of London since September 2017. He was with the Department of Informatics at Kings College London from December 2007 to August 2017, where he was Professor of Wireless Communications from April 2013 to August 2017 and a Visiting Professor from September 2017.

He was an Assistant Professor in the Department

of Electrical and Computer Engineering, National University of Singapore from August 2000 to December 2007. His research interests include 5G Wireless Networks, Internet of Things (IOT) and Molecular Communications. He published nearly 400 technical papers in scientific journals and international conferences. He is a co-recipient of the Best Paper Awards presented at the IEEE International Conference on Communications 2016 (ICC'2016) and IEEE Global Communications Conference 2017 (GLOBECOM'2017). He is an IEEE Distinguished Lecturer. He has been selected as a Web of Science Highly Cited Researcher in 2016.

He is an Editor for IEEE TRANSACTIONS ON COMMUNICATIONS. He was an Editor for ieEe transactions on Wireless Communications (2006-2011), IEEE TRANSACTIONS ON VEHICULAR TECHNOLOGY (2006-

2017), IEEE Wireless Communications Letters and IEEE Signal Processing LetTeRs. He served as the Chair for the Signal Processing and Communication Electronics Technical Committee of IEEE Communications Society and Technical Program Chair and member of Technical Program Committees in numerous IEEE conferences. He received the IEEE Communications Society SPCE outstanding service award 2012 and IEEE Communications Society RCC outstanding service award 2014.

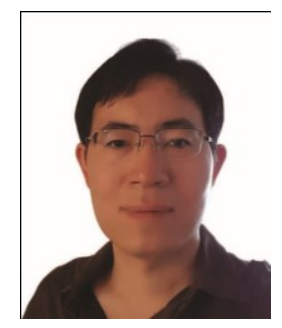

Byonghyo Shim (S'95-M'97-SM'09) received the B.S. and M.S. degrees in control and instrumentation engineering from Seoul National University, South Korea, in 1995 and 1997, respectively, and the M.S. degree in mathematics and the Ph.D. degree in electrical and computer engineering from the University of Illinois at Urbana-Champaign, IL, USA, in 2004 and

2005, respectively. From 1997 and 2000, he was an Officer (First Lieutenant) and also an Academic Fulltime Instructor with the Department of Electronics

Engineering, Korean Air Force Academy. From 2005 to 2007, he was a Staff Engineer with Qualcomm Inc., San Diego, CA, USA. From 2007 to 2014, he was an Associate Professor with the School of Information and Communication, Korea University, Seoul. Since 2014, he has been with the Seoul National University, where he is currently a Professor with the Department of Electrical and Computer Engineering. His research interests include wireless communications, statistical signal processing, compressed sensing, and machine learning. He is an elected member of the Signal Processing for Communications and Networking Technical Committee of the IEEE Signal Processing Society. He was a recipient of the M. E. Van Valkenburg Research Award from University of Illinois in 2005, the

Hadong Young Engineer Award from the IEIE in 2010, the Irwin Jacobs Award from Qualcomm and KICS in 2016, and the Shinyang research award from SNU in 2017. He has served as an Associate Editor of the IEEE Transactions on Signal Processing, the IEEE TRANSACtions on COMMUNICATIONS, the IEEE WIRELESS COMMUNICATIONS LETTERS, and the JOURNAL OF COMmUnications and NetWorks, and a Guest Editor of the IEEE Journal on Selected AREAS in Communications. 